\title{
ADDITIONS TO THE LICHEN BIOTA OF SE SIBERIA: RECORDS FROM THE STANOVOYE NAGOR'E HIGHLANDS (TRANS-BAIKAL REGION, RUSSIA)
}

\author{
Sergey Chesnokov ${ }^{1} \&$ Lydmila Konoreva $^{2}$
}

\begin{abstract}
This paper reports 120 species of lichens and one lichenicolous fungus from the Stanovoye Nagor'e Highlands in southeastern Siberia, including 64 species new for the area. Some of the newly recorded species are extremely rare in Russia [e.g., Bryonora curvescens (Mudd) Poelt, Gyalideopsis alnicola Noble \& Vězda and Pilophorus strumaticus Nyl. ex Cromb.]. The distribution and habitat preferences of several rare species are briefly discussed.
\end{abstract}

Key words: Asia, biodiversity, distribution, lichenized Ascomycota, mountain tundra, rare species, taiga

Sergey Chesnokov, Komarov Botanical Institute, Prof. Popov Str., 2, St. Petersburg, 197376, Russia; e-mail: lukinbrat@mail.ru Lydmila Konoreva, Komarov Botanical Institute, Prof. Popov Str., 2, St. Petersburg, 197376, Russia, and Polar-Alpine Botanical Garden and Institute, Kirovsk, 184256,Russia; e-mail: ajdarzapov@yandex.ru

\section{INTRODUCTION}

The Stanovoye Nagor'e Highlands are situated in southeastern Siberia, NE of Lake Baikal (Fig. 1). The area is a complex of mountain systems separated by narrow intermountain depressions. The Kodar Ridge is the highest part of the Stanovoye Nagor'e Highlands, with summits higher than $2700 \mathrm{~m}$ a.s.l. (the highest summit is $3071 \mathrm{~m}$ a.s.1.) (Kulakov et al. 2002). This region is an area of contact between several types of biota (Makryi 1999). The vegetation of the area is highly diverse, with taiga forest, subalpine thin forest, and mountain tundra. Among the vegetation zones, mountain tundra is the most important for lichen biodiversity.

Southeastern Siberia, which includes the Stanovoye Nagor'e Highlands, are considered the richest part of Russia in terms of number of lichen species (Makryi 1999), but the lichens of the Stanovoye Nagor'e Highlands are poorly investigated. Currently 569 lichen species are known from the area (Makryi 1999, 2002, 2005, 2012, 2013, 2014; Anonymous 2008; Bardunov et al. 2005; Konoreva 2013; Konoreva \& Andreev 2013a, b;

\footnotetext{
1 Corresponding author
}

Lishtva et al. 2013; Gerasimova et al. 2014). For comparison, 1054 lichen species are known from the Sayany Mountains, and 571 from Altaisky Krai (Sedel'nikova 2001; Davydov 2014). The lichen biota of the Stanovoye Nagor'e Highlands was studied by V. Burkova in 1963-1969 but her materials were not published. More recently, her collection was studied by T. Makryi, who reported many new and rare species for Asia and Russia (Makryi 1999, 2002, 2005, 2012, 2013, 2014). The Vitimsky Nature Reserve, located in the Kodar range of the Stanovoye Nagor'e Highlands, were the subject of a detailed lichenological survey by Bardunov et al. (2005).

The present paper is based on lichenological materials collected during several expeditions to the Stanovoye Nagor'e Highlands in 2011-2014. The main purpose of the study was to contribute to the knowledge of the lichen diversity of the area. Some preliminary results were published by Konoreva (2013), Konoreva and Andreev (2013a, b) and Gerasimova et al. (2014). Here we list all the lichens recognized in the area, and briefly discuss the habitat preferences and distribution of several rare species. 


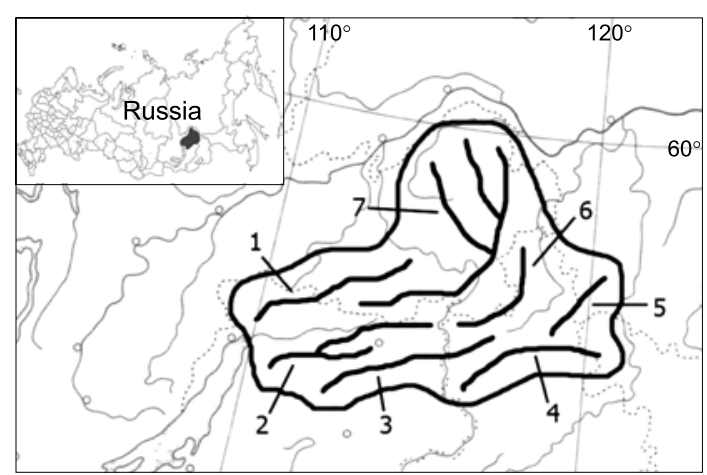

Fig. 1. The mountain systems of the Stanovoye Nagor'e Highlands: 1 - Verkhneangarsky Ridge, 2 - Severo-Muyskiy Ridge, 3 - Yuzhno-Muyskiy Ridge, 4 - Kalar Ridge, 5 - Udokan Ridge, 6 - Kodar Ridge, 7 - Delyun-Uransky Ridge.

\section{MATERIAL AND METHODS}

We collected the material in the eastern part of the Stanovoye Nagor'e Highlands, from sites at 800-2400 m a.s.l. in the following ecosystems: mountain tundra, subalpine light forest, taiga forest, and nival community near snowfields and glacier.

List of collecting sites (WGS 84 system was used for geographical GPS coordinates):

1 - KODAR RIDGE, area near Leprindinskie lakes, $56^{\circ} 39^{\prime} 26.8^{\prime \prime} \mathrm{N}, 117^{\circ} 25^{\prime} 33.6^{\prime \prime} \mathrm{E}, 1698 \mathrm{~m}$ a.s.l., stony placers with large boulders, 14 Aug. 2012.

2 - KODAR RIDGE, area near Leprindinskie lakes, $56^{\circ} 39^{\prime} 35.5^{\prime \prime} \mathrm{N}, 117^{\circ} 25^{\prime} 20.0^{\prime \prime} \mathrm{E}, 1721 \mathrm{~m}$ a.s.l., near creek, 14 Aug. 2012.

3 - KODAR RIDGE, area near Leprindinskie lakes, $56^{\circ} 39^{\prime} 41.0^{\prime \prime} \mathrm{N}, 117^{\circ} 25^{\prime} 12.3^{\prime \prime} \mathrm{E}, 1784 \mathrm{~m}$ a.s.l., stony placers with large boulders, 14 Aug. 2012.

4 - KODAR RIDGE, Leprindinskoe plateau, southern slope, 56 $39^{\prime} 36^{\prime \prime} \mathrm{N}, 117^{\circ} 25^{\prime} 17^{\prime \prime} \mathrm{E}, 1716 \mathrm{~m}$ a.s.l., creek, granite boulders, thicket with Salix sp. and Pinus pumila (Pall.) Regel., 14 Aug. 2012.

5 - Kodar Ridge, Leprindinskoe plateau, $56^{\circ} 39^{\prime} 40^{\prime \prime} \mathrm{N}, 117^{\circ} 25^{\prime} 14^{\prime \prime} \mathrm{E}, 1748 \mathrm{~m}$ a.s.l., turfing rocky slope of southern exposure, funnel of creek watershed, 14 Aug. 2012.

6 - KodAr RidGe, Leprindinskoe plateau, southern slope, $56^{\circ} 39^{\prime} 19^{\prime \prime} \mathrm{N}, 117^{\circ} 25^{\prime} 33^{\prime \prime} \mathrm{E}, 1660 \mathrm{~m}$ a.s.l., disintegrated granite boulders, shrub-lichen tundra, 14 Aug. 2012.

7 - KodAr RIDGe, ascent to Leprendinskoe plateau, $56^{\circ} 40^{\prime} 05.3^{\prime \prime} \mathrm{N}, 117^{\circ} 24^{\prime} 50.0^{\prime \prime} \mathrm{E}, 2142 \mathrm{~m}$ a.s.l., mountain tundra, 15 Aug. 2012.
8 - KoDAR RIDGE, ascent to Leprendinskoe plateau, $56^{\circ} 40^{\prime} 0.9^{\prime \prime} \mathrm{N}, 117^{\circ} 24^{\prime} 52.4^{\prime \prime} \mathrm{E}, 2042 \mathrm{~m}$ a.s.1., near snowfield, 15 Aug. 2012.

9 - KoDAR RIDGE, Leprindinskoe plateau, southern slope, $56^{\circ} 40^{\prime} 04^{\prime \prime} \mathrm{N}, 117^{\circ} 24^{\prime} 49^{\prime \prime} \mathrm{E}, 2128 \mathrm{~m}$ a.s.l., rocks and broken stones in streambed, 15 Aug. 2012.

10 - Kodar Ridge, Leprindinskoye plateau, $56^{\circ} 40^{\prime} 15.6^{\prime \prime} \mathrm{N}, 117^{\circ} 25^{\prime} 17.6^{\prime \prime} \mathrm{E}, 2311 \mathrm{~m}$ a.s.1., mountain tundra, 15 Aug. 2012.

11 - KodAr RIDGE, Leprindinskoe plateau, southern slope, $56^{\circ} 40^{\prime} 00^{\prime \prime} \mathrm{N}, 117^{\circ} 24^{\prime} 52^{\prime \prime} \mathrm{E}, 2041 \mathrm{~m}$ a.s.l., Carex sp.-Salix sp.-Dryas octopetala tundra with stones, 15 Aug. 2012.

12 - Kodar Ridge, Leprindinskoye plateau, $56^{\circ} 40^{\prime} 15.5^{\prime \prime} \mathrm{N}, 117^{\circ} 24^{\prime} 49.1^{\prime \prime} \mathrm{E}, 2299$ m a.s.1., Salix sp.lichen tundra with cereals, 15 Aug. 2012.

13 - KODAR RIDGE, area near Leprindinskie lakes, left side of valley, $56^{\circ} 39^{\prime} 26.6^{\prime \prime} \mathrm{N}, 117^{\circ} 26^{\prime} 0.8^{\prime \prime} \mathrm{E}, 1818 \mathrm{~m}$ a.s.1., top ridge of rocks, 16 Aug. 2012.

14 - KODAR RIDGE, area near Leprindinskie lakes, $56^{\circ} 39^{\prime} 28.3^{\prime \prime} \mathrm{N}, 117^{\circ} 25^{\prime} 49.4^{\prime \prime} \mathrm{E}, 1720 \mathrm{~m}$ a.s.l., left side of valley, rocky outcrops, 16 Aug. 2012.

15 - KodAr Ridge, Leprindinskoe plateau, $56^{\circ} 39^{\prime} 26^{\prime \prime} \mathrm{N}, 117^{\circ} 26^{\prime} 00^{\prime \prime} \mathrm{E}, 1804 \mathrm{~m}$ a.s.l., left bank of stream, slope after fire, granite outcrops, 16 Aug. 2012.

16 - Kodar Ridge, Leprindinskoe plateau, $56^{\circ} 39^{\prime} 26^{\prime \prime} \mathrm{N}, 117^{\circ} 25^{\prime} 57^{\prime \prime} \mathrm{E}, 1760 \mathrm{~m}$ a.s.l., left bank of stream, slope after fire, rocky lichen community with Ledum palustre L. and Betula nana L., 16 Aug. 2012.

17 - KodAr RIDGE, Leprindinskoe plateau, western slope, $56^{\circ} 39^{\prime} 28^{\prime \prime} \mathrm{N}, 117^{\circ} 25^{\prime} 48^{\prime \prime} \mathrm{E}, 1678 \mathrm{~m}$ a.s.l., overgrown rocks, moss-Carex sp. communities, 16 Aug. 2012.

18 - Kodar Ridge, Leprindo Lake, north shore, $56^{\circ} 38^{\prime} 12^{\prime \prime} \mathrm{N}, 117^{\circ} 23^{\prime} 51^{\prime \prime} \mathrm{E}, 1150 \mathrm{~m}$ a.s.1., deep shady cleft in granite rock, stream and waterfall, wet mossy rocks, 17 Aug. 2012.

19 - KodAr Ridge, Leprindo Lake, $56^{\circ} 38^{\prime} 15^{\prime \prime} \mathrm{N}$, $117^{\circ} 23^{\prime} 49^{\prime \prime} \mathrm{E}, 1171 \mathrm{~m}$ a.s.l., north shore with stream and waterfall, deep shady valley surrounded by steep wet granite rocks, depression under waterfall, southern exposure, 17 Aug. 2012.

20 - KodAr Ridge, Leprindo Lake, $56^{\circ} 38^{\prime} 13^{\prime \prime} \mathrm{N}$, $117^{\circ} 23^{\prime} 52^{\prime \prime} \mathrm{E}, 1177 \mathrm{~m}$ a.s.l., north shore with stream and waterfall, deep shady valley surrounded by steep wet granite rocks, steep slopes overgrown with moss, 17 Aug. 2012.

21 - KodAr Ridge, near Maloe Leprindo lake, $56^{\circ} 38^{\prime} 12.0^{\prime \prime} \mathrm{N}, 117^{\circ} 23^{\prime} 48.4^{\prime \prime} \mathrm{E}, 1136 \mathrm{~m}$ a.s.1., waterfall, moss-covered boulders in canyon, 17 Aug. 2012.

22 - UdDKAN Ridge, off spur of Mednaya Moun- 
tain, $56^{\circ} 41^{\prime} 27.7^{\prime \prime} \mathrm{N}, 118^{\circ} 18^{\prime} 48.6^{\prime \prime} \mathrm{E}, 1216 \mathrm{~m}$ a.s.1., stone placer, 19 Aug. 2012.

23 - UdDOKAN RIDGE, off spur of Mednaya Mountain, $56^{\circ} 41^{\prime} 36.9^{\prime \prime} \mathrm{N}, 118^{\circ} 19^{\prime} 08.2^{\prime \prime} \mathrm{E}, 1120 \mathrm{~m}$ a.s.l., thicket of Pinus pumila, stone placer, 19 Aug. 2012.

24 - YuZHNO-MuYskiY Ridge, rocks on bank of Vitim river, $56^{\circ} 12^{\prime} 29.9^{\prime \prime} \mathrm{N}, 115^{\circ} 44^{\prime} 57.3^{\prime \prime} \mathrm{E}, 496 \mathrm{~m}$ a.s.1., Pinus sylvestris forest passing into Alnus sp. forest with Betula sp., 23 Aug. 2012.

25 - Kodar Ridge, confluence of Medvezhy and Surprizniy creeks, $56^{\circ} 54^{\prime} 55.6^{\prime \prime} \mathrm{N}, 117^{\circ} 37^{\prime} 40.7^{\prime \prime} \mathrm{E}$, $1667 \mathrm{~m}$ a.s.l., forest tundra, 1 July 2013.

26 - KodAr RidGe, confluence of Medvezhy and Surpriznii creeks, $56^{\circ} 54^{\prime} 54.0^{\prime \prime} \mathrm{N}, 117^{\circ} 37^{\prime} 40.8^{\prime \prime} \mathrm{E}, 1678 \mathrm{~m}$ a.s.1., rock on northern slopes, 1 July 2013.

27 - Kodar Ridge, Medvezhy brook valley, $56^{\circ} 54^{\prime} 40.3^{\prime \prime} \mathrm{N}, 117^{\circ} 37^{\prime} 04.2^{\prime \prime} \mathrm{E}, 1764 \mathrm{~m}$ a.s.l., site of tundra with Betula nana, 1 July 2013.

28 - Kodar Ridge, Medvezhy brook valley, $56^{\circ} 54^{\prime} 11.2^{\prime \prime} \mathrm{N}, 117^{\circ} 34^{\prime} 55.5^{\prime \prime} \mathrm{E}, 1990 \mathrm{~m}$ a.s.l., nival tundra, 1 July 2013.

29 - Kodar Ridge, Medvezhy brook valley, $56^{\circ} 54^{\prime} 35.8^{\prime \prime} \mathrm{N}, 117^{\circ} 36^{\prime} 42.1^{\prime \prime} \mathrm{E}, 1796 \mathrm{~m}$ a.s.l., stone rubble, 1 July 2013.

30 - KodAr RidGe, Pionerskiy creek, $56^{\circ} 54^{\prime} 29.3^{\prime \prime} \mathrm{N}$, $117^{\circ} 36^{\prime} 00.0^{\prime \prime} \mathrm{E}, 1805 \mathrm{~m}$ a.s.1., 1 July 2013.

31 - KoDAR RIDGE, Baltiyskoe gorge, 56 $54^{\prime} 19.9^{\prime \prime} \mathrm{N}$, $117^{\circ} 39^{\prime} 20.9^{\prime \prime} \mathrm{E}, 1875 \mathrm{~m}$ a.s.l., creek and large boulders, 2 July 2013.

32 - KodAr RidGe, Baltiyskoe gorge, 56 $53^{\prime} 46.5^{\prime \prime} \mathrm{N}$, $117^{\circ} 39^{\prime} 12.4^{\prime \prime} \mathrm{E}, 1964 \mathrm{~m}$ a.s.l., stony placers, 2 July 2013.

33 - KoDAR RIDGE, Baltiyskoe gorge, $56^{\circ} 54^{\prime} 18.6^{\prime \prime} \mathrm{N}$, $117^{\circ} 39^{\prime} 18.9^{\prime \prime} \mathrm{E}, 1918 \mathrm{~m}$ a.s.l., stone rubble, 2 July 2013.

34 - KodAr RidGe, Baltiyskoe gorge, $56^{\circ} 55^{\prime} 13.8^{\prime \prime} \mathrm{N}$, $117^{\circ} 39^{\prime} 19.8^{\prime \prime} \mathrm{E}, 1727 \mathrm{~m}$ a.s.l., slope with Pinus pumila, 2 July 2013.

35 - Kodar Ridge, Mramorny settlement, camp point "Gora", 56 $54^{\prime} 18.7^{\prime \prime} \mathrm{N}, 117^{\circ} 42^{\prime} 31.5^{\prime \prime} \mathrm{E}, 1877 \mathrm{~m}$ a.s.l., stone rubble, 3 July 2013.

36 - KodAr Ridge, Mramornoe gorge, Mramorny settlement, $56^{\circ} 54^{\prime} 37.6^{\prime \prime} \mathrm{N}, 117^{\circ} 42^{\prime} 29.4^{\prime \prime} \mathrm{E}, 1806 \mathrm{~m}$ a.s.l., 3 July 2013.

37 - KodAR RidGe, Mramornoe gorge, $56^{\circ} 54^{\prime} 17.4^{\prime \prime} \mathrm{N}$, $117^{\circ} 42^{\prime} 33.5^{\prime \prime}$ E, 1885 m a.s.l., near creek, 3 July 2013.

38 - KODAR RidGE, Surpriznoe lake, $56^{\circ} 54^{\prime} 20.5^{\prime \prime} \mathrm{N}$, $117^{\circ} 38^{\prime} 20.3^{\prime \prime}$ E, $2011 \mathrm{~m}$ a.s.1., mountain tundra, 5 July 2013.

39 - Kodar Ridge, Zolotoy brookvalley, $56^{\circ} 55^{\prime} 53.8^{\prime \prime} \mathrm{N}, 117^{\circ} 36^{\prime} 37.8^{\prime \prime} \mathrm{E}, 1957$ m a.s.1., 6 July 2013.

40 - KodAR Ridge, Zolotoy brook valley, $56^{\circ} 55^{\prime} 56.8^{\prime \prime} \mathrm{N}, 117^{\circ} 36^{\prime} 50.7^{\prime \prime} \mathrm{E}, 1900 \mathrm{~m}$ a.s.l., tundra with Salix sp. and Rhododendron aureum Georgi, 6 July 2013.
41 - Kodar Ridge, Zolotoy brook valley, $56^{\circ} 55^{\prime} 57.4^{\prime \prime} \mathrm{N}, 117^{\circ} 36^{\prime} 51.5^{\prime \prime} \mathrm{E}, 1857 \mathrm{~m}$ a.s.1., rocks, 6 July 2013.

42 - Kodar Ridge, Zolotoy brook valley, $56^{\circ} 56^{\prime} 09.1^{\prime \prime} \mathrm{N}, 117^{\circ} 37^{\prime} 18.0^{\prime \prime} \mathrm{E}, 1619 \mathrm{~m}$ a.s.1., northwestern slope, 6 July 2013.

43 - KodAr RidGe, left bank of Sredniy Sakukan, opposite Exa gorge, 56 $55^{\prime} 00.9^{\prime \prime} \mathrm{N}, 117^{\circ} 48^{\prime} 57.8^{\prime \prime} \mathrm{E}$, $1175 \mathrm{~m}$ a.s.l., forest with large boulders, 7 July 2013.

44 - Kodar Ridge, Anarga river, left bank, $56^{\circ} 55^{\prime} 10.6^{\prime \prime} \mathrm{N}, 118^{\circ} 01^{\prime} 45.1^{\prime \prime} \mathrm{E}, 941 \mathrm{~m}$ a.s.1., rocks, 9 July 2013.

45 - Kodar Ridge, Anarga river, left bank, $56^{\circ} 55^{\prime} 19.4^{\prime \prime} \mathrm{N}, 118^{\circ} 01^{\prime} 50.0^{\prime \prime} \mathrm{E}, 1071 \mathrm{~m}$ a.s.l., rocks, 9 July 2013.

46 - KodAr RidGe, first canyon of creek west of Anarga river, $56^{\circ} 55^{\prime} 09.9^{\prime \prime} \mathrm{N}, 118^{\circ} 00^{\prime} 04.3^{\prime \prime} \mathrm{E}, 1592 \mathrm{~m}$ a.s.1., rocks, 10 July 2013.

47 - KODAR RIDGE, first canyon of creek west of Anarga river, $56^{\circ} 54^{\prime} 38.9^{\prime \prime} \mathrm{N}, 18^{\circ} 00^{\prime} 49.4^{\prime \prime} \mathrm{E}, 1016 \mathrm{~m}$ a.s.l., rocks, 10 July 2013.

48 - KODAR RidGe, left bank of Sredniy Sakukan, 56 54'47.9"N, 11750'29.5"E, 1049 m a.s.1., Populus suaveolens-Betula platyphylla-Larix gmelinii forest, 4 June 2014.

49 - KodAr RidGe, Shan'go valley, left bank, $56^{\circ} 57^{\prime} 22.3^{\prime \prime} \mathrm{N}, 117^{\circ} 48^{\prime} 23.2^{\prime \prime} \mathrm{E}, 1708 \mathrm{~m}$ a.s.l., thickets of Salix sp. and Pinus pumila near rock, 6 June 2014.

50 - Kodar Ridge, bank of Shan'go lake, $56^{\circ} 58^{\prime} 41.4^{\prime \prime} \mathrm{N}, 117^{\circ} 47^{\prime} 50.3^{\prime \prime} \mathrm{E}, 1908 \mathrm{~m}$ a.s.1., lichen tundra, 7 June 2014.

51 - KoDAR RIDGE, end of Shan'go valley, hill opposite ' 60 let USSR' pass, $56^{\circ} 58^{\prime} 24.9^{\prime \prime} \mathrm{N}, 117^{\circ} 49^{\prime} 01.8^{\prime \prime} \mathrm{E}$, $1863 \mathrm{~m}$ a.s.l., south slope, mountain tundra, 8 June 2014.

52 - Kodar RIDGE, end of Shan'go valley, hill opposite ' 60 let USSR' pass, $56^{\circ} 58^{\prime} 35.6^{\prime \prime} \mathrm{N}, 117^{\circ} 49^{\prime} 11.9^{\prime \prime} \mathrm{E}$, $1994 \mathrm{~m}$ a.s.l., lichen tundra, 8 June 2014.

53 - KodAR RIDGE, end of Shan'go valley, hill opposite ' 60 let USSR' pass, $56^{\circ} 58^{\prime} 29.6^{\prime \prime} \mathrm{N}, 117^{\circ} 49^{\prime} 11.8^{\prime \prime} \mathrm{E}$, $1928 \mathrm{~m}$ a.s.l., south slope, Cassiope sp. tundra, 8 June 2014.

54 - Kodar Ridge, end of Shan'go valley, $56^{\circ} 58^{\prime} 39.3^{\prime \prime} \mathrm{N}, 117^{\circ} 49^{\prime} 10.9^{\prime \prime} \mathrm{E}, 2187 \mathrm{~m}$ a.s.l., hill opposite ' 60 let USSR' pass, western slope, 8 June 2014.

55 - Kodar Ridge, end of Shan'go valley, $56^{\circ} 58^{\prime} 37.2^{\prime \prime} \mathrm{N}, 117^{\circ} 49^{\prime} 12.8^{\prime \prime} \mathrm{E}, 2037 \mathrm{~m}$ a.s.l., hill opposite ' 60 let USSR' pass, mountain tundra with large boulders, 8 June 2014.

56 - KodAr Ridge, Shan'go valley, right bank, $56^{\circ} 57^{\prime} 31.4^{\prime \prime} \mathrm{N}, 117^{\circ} 48^{\prime} 13.3^{\prime \prime} \mathrm{E}, 1715 \mathrm{~m}$ a.s.l., light forest with Larix gmelinii (Rupr.) Kuzen. on border with tundra, 9 June 2014. 
57 - Kodar Ridge, Shan'go valley, left bank, $56^{\circ} 57^{\prime} 30.4^{\prime \prime} \mathrm{N}, 117^{\circ} 48^{\prime} 19.7^{\prime \prime} \mathrm{E}, 1720 \mathrm{~m}$ a.s.l., stones in creek, moss-covered bank of stream, 9 June 2014.

58 - Kodar Ridge, Shan'go valley, left bank, $56^{\circ} 57^{\prime} 15.7^{\prime \prime} \mathrm{N}, 117^{\circ} 48^{\prime} 21.4^{\prime \prime} \mathrm{E}, 1763 \mathrm{~m}$ a.s.1., Larix gmelinii-moss-lichen forest, 9 June 2014.

59 - Kodar Ridge, Shan'go valley, left bank, $56^{\circ} 56^{\prime} 21.6^{\prime \prime} \mathrm{N}, 117^{\circ} 48^{\prime} 33.7^{\prime \prime} \mathrm{E}, 1679 \mathrm{~m}$ a.s.l., Betula sp.Larix gmelinii forest with Salix sp. and Pinus pumila near creek, 10 June 2014.

60 - KodAr Ridge, Shan'go valley, left bank, $56^{\circ} 56^{\prime} 21.4^{\prime \prime} \mathrm{N}, 117^{\circ} 48^{\prime} 38.6^{\prime \prime} \mathrm{E}, 1726 \mathrm{~m}$ a.s.l., rocky outcrops on slopes, large boulders, 10 June 2014.

61 - Kodar Ridge, Shan'go valley, left bank, $56^{\circ} 56^{\prime} 38.0^{\prime \prime} \mathrm{N}, 117^{\circ} 48^{\prime} 17.0^{\prime \prime} \mathrm{E}, 1634 \mathrm{~m}$ a.s.1., Larix gmelinii-green moss forest, 10 June 2014.

62 - KODAR RIDGE, Azarova glacier, $56^{\circ} 53^{\prime} 58.1^{\prime \prime} \mathrm{N}$, $117^{\circ} 34^{\prime} 59.2^{\prime \prime}$ E, 1947 m a.s.l., moraine, 13 June 2014.

63 - KodAR RIDGE, Medvezhy brook valley, right bank, 56 $54^{\prime} 51.7^{\prime \prime N}, 117^{\circ} 37^{\prime} 45.9^{\prime \prime}$ E, $1792 \mathrm{~m}$ a.s.l., Surprizniy creek, thickets of Pinus pumila, 14 June 2014.

64 - Kodar Ridge, Medvezhy brook valley, left bank, $56^{\circ} 54^{\prime} 47.0^{\prime \prime} \mathrm{N}, 117^{\circ} 37^{\prime} 01.7^{\prime \prime} \mathrm{E}, 1779 \mathrm{~m}$ a.s.1., stones among thickets of Pinus pumila, 14 June 2014.

65 - KodAr RidGe, Medvezhy brook valley, bank, $56^{\circ} 54^{\prime} 46.1^{\prime \prime} \mathrm{N}, 117^{\circ} 37^{\prime} 04.5^{\prime \prime} \mathrm{E}, 1752 \mathrm{~m}$ a.s.l., moss-lichen tundra with Pinus pumila; 14 June 2014.

66 - KodAR RIDGE, confluence of Uglovoy creek and Sredniy Sakukan, $56^{\circ} 56^{\prime} 33.0^{\prime \prime} \mathrm{N}, 117^{\circ} 36^{\prime} 50.7^{\prime \prime} \mathrm{E}$, $1670 \mathrm{~m}$ a.s.1., rocky outcrops, 15 June 2014.

67 - KODAR RIDGE, source of Sredniy Sakukan river, $56^{\circ} 57^{\prime} 58.9^{\prime \prime} \mathrm{N}, 117^{\circ} 37^{\prime} 59.6^{\prime \prime} \mathrm{E}, 1925 \mathrm{~m}$ a.s.1., mountain tundra with large boulders, 16 June 2014.

68 - Kodar Ridge, Uglovoy creek, left bank, $56^{\circ} 56^{\prime} 38.6^{\prime \prime} \mathrm{N}, 117^{\circ} 36^{\prime} 43.7^{\prime \prime} \mathrm{E}, 1742 \mathrm{~m}$ a.s.l., slope with Rhododendron aureum and Betula nana, 16 June 2014.

69 - KodAr RidGe, Uglovoe lake, $56^{\circ} 56^{\prime} 51.8^{\prime \prime} \mathrm{N}$, $117^{\circ} 34^{\prime} 58.1^{\prime \prime E}, 1916 \mathrm{~m}$ a.s.1., rocks, 17 June 2014.

70 - KodAr Ridge, Carskiy Tron peak, shaded gorge, $56^{\circ} 56^{\prime} 55.7^{\prime \prime} \mathrm{N}, 117^{\circ} 38^{\prime} 02.0^{\prime \prime} \mathrm{E}, 1993 \mathrm{~m}$ a.s.1., nival habitat, 18 June 2014.

71 - KodAr RidGe, Sredniy Sakukan valley, right bank, 56 $56^{\prime} 28.2^{\prime \prime} \mathrm{N}, 117^{\circ} 36^{\prime} 55.3^{\prime \prime} \mathrm{E}, 1686 \mathrm{~m}$ a.s.1., Salix sp.-herb-green moss community with creek, 18 June 2014.

72 - Kodar Ridge, Sredniy Sakukan valley, left bank, $56^{\circ} 53^{\prime} 54.7^{\prime \prime} \mathrm{N}, 117^{\circ} 57^{\prime} 48.1^{\prime \prime} \mathrm{E}, 884 \mathrm{~m}$ a.s.1., Larix gmelinii forest, 20 June 2014.

The material was identified by standard methods. For identification of lichen species we used different keys, monographs and other studies (Ahti \& Stenroos
2013; Andreev et al. 2003, 2008; Brodo et al. 2001; Dombrovskaya 1996; Foucard 2001; Lücking et al. 2006; Øvstedal et al. 2009; Spribille et al. 2014; Smith et al. 2009).

The samples are kept in the Herbarium of the Komarov Botanical Institute (LE) and the Polar-Alpine Botanical Garden and Institute (KPABG).

Lichen species new for the Stanovoye Nagor'e Highlands are indicated with '!'. One lichenicolous fungus is asterisked.

\section{RESULTS AND DISCUSSION}

In this study we recorded 120 lichen species and one lichenicolous fungus from the Stanovoye Nagor'e Highlands. Most of the species are lignicolous, corticolous or muscicolous; the saxicolous group was least represented. Sixty-four of the recorded species are new for the area. They include a number of taxa very rare in the country, including Bryonora curvescens, Gyalideopsis alnicola, Gyalolechia lenae, Pilophorus strumaticus, Phaeophyscia dissecta, Ph. endococcinodes, Ph. hirtella, Psora vallesiaca, Rhizocarpon cinereonigrum, Squamarina cartilaginea, Stereocaulon wrightii and Xylographa trunciseda; the world distribution and habitat preferences of these species are discussed briefly below. The study also yielded further regional records of lichens new for particular ridges in the Stanovoye Nagor'e Highlands, such as the Kodar Ridge (103 species), YuzhnoMuyskiy Ridge (13 species) and Udokan Ridge (11 species).

\section{"! Abrothallus parmeliarum (Sommerf.) Arnold}

Collecting site: 44, 45 - on thalli of Parmelia sulcata Tayl. and Melanohalea olivacea (L.) O. Blanco et al.

\section{Absconditella lignicola Vězda \& Pišut}

Collecting site: 44 - on bark of Betula sp.

Notes. Species new for Kodar Ridge. Previously reported from Kalar Ridge by Konoreva and Andreev (2013b).

Amandinea punctata (Hoffm.) Coppins \& Scheid.

Collecting SITE: 44, 45, 65 - on bark of Betula sp., Sorbus sibirica Hedl., Pinus pumila (Pall.) Regel and rotten wood. 
Notes. Species new for Kodar Ridge. Previously reported from Delyun-Uransky Ridge by Bardunov et al. (2005) and Makryi (2002) as Buellia stigmatea (Schaer.) Körb.

Amygdalaria panaeola (Ach.) Hertel \& Brodo Collecting site: 7, 22, 38 - on stone.

Notes. Species new for Udokan Ridge. Previously reported from Delyun-Uransky and Kodar Ridges by Bardunov et al. (2005) and Makryi (2002) respectively.

\section{Arctoparmelia centrifuga (L.) Hale}

Collecting Site: $1,10,22,31,38,41-$ on stone and soil on stone.

Notes. Species new for Udokan Ridge. Previously reported from Delyun-Uransky and Kodar Ridges by Bardunov et al. (2005) and Makryi (2002) respectively.

Arthonia radiata (Pers.) Ach.

Collecting Site: 44 - on bark of Sorbus sibirica.

Notes. Species new for Kodar Ridge. Previously reported from Delyun-Uransky Ridge by Bardunov et al. (2005).

Athallia cerinelloides (Erichsen) Arup et al.

Collecting site: 45, 46 - on bark Populus tremula L. and lignum.

Notes. Species new for Kodar Ridge. Previously reported from Udokan Ridge by Konoreva and Andreev (2013b) as Caloplaca cerinelloides (Erichsen) Poelt.

Athallia holocarpa (Hoffm.) Arup et al.

Collecting SITE: 44, 46 - on stone.

Notes. Species new for Kodar Ridge. Previously reported from Delyun-Uransky Ridge as $\mathrm{Ca}$ loplaca holocarpa (Hoffm. ex. Ach.) A. E. Wade (Bardunov et al. 2005).

\section{!Bacidia subincompta (Nyl.) Arnold}

Collecting Site: 44 - on bark of Betula sp.

!Biatorella conspurcans Norman

Collecting site: 44 - on lignum.
!Bilimbia cf. microcarpa (Th. Fr.) Th. Fr.

Collecting SITE: 32 - on mosses on stone.

!Blastenia ammiospila (Wahlenb.) Arup et al.

Collecting site: $36,42,62,71$ - on plant debris, mosses and the bark and wood of Salix sp.

!Bryonora curvescens (Mudd) Poelt

Collecting Site: 32,41 - on mosses.

Notes. This is the second record of this species in Russia. Previously it was reported from Taimyr (Siberian Arctic) (Øvstedal et al. 2009; Kristinsson et al. 2010; Urbanavichus 2010). It is an arcticalpine species, occurring in Europe, Greenland, Iceland, Macaronesia, North and South America (Smith et al. 2009).

!Buellia epigaea (Pers.) Tuck.

Collecting Site: 41,49 - on soil and plant debris.

Calicium glaucellum Ach.

Collecting site: 43, 48 - on bark of Salix sp. and rotten wood.

Notes. Species new for Kodar Ridge. Previously reported from Udokan Ridge by Konoreva and Andreev (2013b).

Calicium trabinellum (Ach.) Ach.

Collecting Site: 20, 36, 48, 56, 58 - on bark and wood of Larix gmelinii and rotten wood.

Notes. Species new for Kodar Ridge. Previously reported from Delyun-Uransky Ridge by Bardunov et al. (2005).

!Caloplaca saxicola (Hoffm.) Nordin s.l.

Collecting Site: 24, 44, 45, 47 - on stone.

Cetraria sepincola (Ehrh.) Ach.

Collecting site: 2, 36, 63, 65, 68, 71 - on branches of Betula nana, lignum, bark of Pinus pumila and bark and branches of Salix sp.

Notes. Species new for Kodar Ridge. Previously reported from Delyun-Uransky Ridge by Bardunov et al. (2005).

Cetrelia cetrarioides (Delise ex Duby) W. L. Culb. \& C. F. Culb. 
Collecting site: 43,44 - on mossy stone and bark of Sorbus sibirica Hedl.

Notes. Species new for Kodar Ridge. Previously reported from Delyun-Uransky Ridge by Bardunov et al. (2005).

!Cetrelia olivetorum (Nyl.) W. L. Culb. \& C. F. Culb.

COLLeCting site: 20,21,38, 44 - on mossy stone, bark of Betula ermanii Cham and Sorbus sibirica, and lignum.

Cladonia gracilis (L.) Willd.

Collecting site: 1, 2, 3, 7, 9, 11, 16, 24, 36, 41, 50, $51,52,64,65$ - on soil, mosses and lignum.

Notes. Species new for Yuzhno-Muyskiy Ridge. Previously reported from Kodar (Bardunov et al. 2005; Konoreva \& Andreev 2013a, b), Delyun-Uransky (Bardunov et al. 2005) and Kalar Ridges (Konoreva \& Andreev 2013b).

Cladonia pyxidata (L.) Hoffm.

Collecting site: $1,3,7,9,13,14,17,24,36,38,41$, $44,45,46,56,66$ - on soil, lignum, mosses and bark of Larix gmelinii.

Notes. Species new for Yuzhno-Muyskiy Ridge. Previously reported from Kodar and Delyun-Uransky Ridges (Bardunov et al. 2005).

Cladonia stygia (Fr.) Ruoss

Collecting site: $25,31,50,51,52,56,61,65,67,70$, 71 - on soil, mosses and soil on stone.

Notes. Species new for Kodar Ridge. Previously reported from Kalar Ridge by Konoreva and Andreev (2013b).

Cladonia verticillata (Hoffm.) Schaer.

COllecting site: 38,53 - on soil.

Notes. Species new for Kodar Ridge. Previously reported from Delyun-Uransky Ridge by Bardunov et al. (2005) as Cladonia cervicornis subsp. verticillata (Hoffm.) Ahti.

!Cystocoleus ebeneus (Dillwyn) Thwaites

COllecting site: 44 - on stone.

Dibaeis baeomyces (L. f.) Rambold \& Hertel

Collecting Site: $30,47,52$ - on soil.
Notes. Species new for Kodar Ridge. Previously reported from Delyun-Uransky Ridge by Bardunov et al. (2005).

!Diplotomma alboatrum (Hoffm.) Flot.

COllecting SITE: 44 - on stone.

!Enchylium tenax (Sw.) Gray

Collecting Site: $19,24,45$ - on stone.

!Fuscopannaria confusa (P. M. Jørg.) Р. М. Jørg. Collecting site: 13,43 - on mosses and soil on stone.

Fuscopannaria praetermissa (Nyl.) P. M. Jørg. Collecting site: 7, 19, 45, 57 - on soil and mosses.

Notes. Species new for Kodar Ridge. Previously reported from Delyun-Uransky Ridge by Bardunov et al. (2005).

Graphis scripta (L.) Ach.

Collecting site: 21, 44, 46 - on bark of Betula ermanii, Sorbus sibirica and rotten branches of Betula sp.

Notes. Species new for Kodar Ridge. Previously reported from Delyun-Uransky and Kalar Ridges by Bardunov et al. (2005) and Konoreva and Andreev (2013b) respectively.

!Gyalideopsis alnicola Noble \& Vězda

Collecting site: 46 - on rotten wood.

Notes. This is the second record of this species in Russia. Previously it was reported from the Leningrad region (Stepanchikova et al. 2013). The species is rare, and reported from Europe (Norway, Italy) and North America (Canada) (Andreev et al. 2008). It was reported from regions with an oceanic climate, where it grows on bark of Alnus sp. and Picea sp. in rainforest. Gyalideopsis alnicola was considered to be a synonym of $G$. piceicola (Nyl.) Vězda \& Poelt by several authors (e.g., Santesson et al. 2004). The most recent studies, however, indicated that they are two separate species (Lücking et al. 2005, 2006).

Gyalolechia flavovirescens (Wulfen) Søchting et al.

Collecting site: 19, 44, 45, 47 - on stone.

Notes. Species new for Kodar Ridge. Previ- 
ously reported from Delyun-Uransky Ridge by Bardunov et al. (2005) as Caloplaca flavovirescens (Wulfen) Dalla Torre \& Sarnth.

!Gyalolechia lenae (Søchting \& G. Figueras) Søchting, Frödén \& Arup

COllecting Site: 44,45 - on base-rich rock.

Notes. This is a rare species in Russia. Previously reported from Jacutia and the Altai Region. Also reported from Mongolia (Søchting \& Figueras 2007). It grows on limestone or base-rich rocks.

Hypocenomyce scalaris (Ach.) M. Choisy

COllecting SITE: 20, 43 - on rotten wood and the bark of Larix gmelinii.

Notes. Species new for Kodar Ridge. Previously reported from Delyun-Uransky Ridge by Bardunov et al. (2005).

!Lecania cyrtellina (Nyl.) Sandst.

Collecting Site: $31,41-$ on plant debris.

!Lecanora albescens (Hoffm.) Branth \& Rostr.

COllecting SITE: 47 - on stone.

!Lecanora boligera (Norman ex Th. Fr.) Hedl.

COllecting site: 41,68 - on plant debris and the bark of Betula nana.

!Lecanora cadubriae (A. Massal.) Hedl.

Collecting Site: 38 - on plant debris.

Lecanora campestris (Schaer.) Hue

Collecting site: $24,44,45,46$ - on stone.

Notes. Species new for Kodar and YuzhnoMuyskiy Ridges. Previously reported from Delyun-Uransky Ridge by Bardunov et al. (2005).

Lecanora circumborealis Brodo \& Vitik.

COLleCting SITE: $34,36,68$ - on bark and branches of Betula nana and rotten wood.

Notes. Species new for Kodar Ridge. Previously reported from Udokan Ridge by Konoreva and Andreev (2013b).

!Lecanora epibryon (Ach.) Ach.

Collecting Site: 9, 13, 44 - on mosses and soil.
Lecanora polytropa (Ehrh. ex Hoffm.) Rabenh.

Collecting site: $3,5,6,8,9,10,12,15,22,27,35$, $38,40,45,50,52-$ on stone.

Notes. Species new for Udokan Ridge. Previously reported from Delyun-Uransky (Bardunov et al. 2005) and Kodar Ridges (Makryi 2002; Bardunov et al. 2005; Konoreva \& Andreev 2013a).

!Lecanora saligna (Schrad.) Zahlbr.

Collecting site: 36,44 - on plant debris, rotten wood and the bark of Sorbus sibirica.

Lecanora symmicta (Ach.) Ach.

Collecting SITE: 44, 45, 48, 63, 65, 71 - on bark of Sorbus sibirica, Pinus pumila, Salix sp. and Betula sp., and rotten wood.

Notes. Species new for Kodar Ridge. Previously reported from Delyun-Uransky (Bardunov et al. 2005), Kalar and Yuzhno-Muyskiy Ridges (Konoreva \& Andreev 2013b).

!Lecidea alpestris Sommerf.

Collecting site: $8,34,36,40,41,42,66$ - on soil and plant debris.

!Lecidea berengeriana (A. Massal.) Th. Fr.

COllecting site: 7, 43 - on mosses and soil.

!Leproplaca cf. chrysodeta (Räsänen) J. R. Laundon COllecting SITE: 47 - on stone.

Leptogium saturninum (Dicks.) Nyl.

Collecting site: $13,21,24,44,45,47,60$ - on bark of Sorbus sibirica and Betula sp., rotten wood, mosses and mossy stone.

Notes. Species new for Kodar Ridge. Previously reported from Yuzhno-Muyskiy (Konoreva \& Andreev 2013b).

Lichenomphalia umbellifera (L.: Fr.) Redhead et al.

Collecting site: 40,46 - on soil and mosses.

Notes. Species new for Kodar Ridge. Previously reported from Delyun-Uransky by Bardunov et al. (2005) as Omphalina umbillifera (L.: Fr.) Quélet. 


\section{!Lobaria amplissima (Scop.) Forssell}

COLLECTING SITE: 44 - on rotten branches.

Notes. The species forms tiny, blackish, densely shrubby cephalodia. They can become free-living and were even named a separate taxon -Dendriscocaulon umhausense (Auersw.) Degel. According to Jørgensen (1998) and Tonsberg and Goward (2001), 'Dendriscocaulon' is the cyanobacterial phototype or 'cyanotype' of foliose lichens containing green algae. We found only 'cyanotypes' of Lobaria amplissima.

\section{Lobaria retigera (Bory) Trevis.}

Collecting site: 44, 46 - on mossy stone.

Notes. The species is red-listed in the Russian Federation (Anonymous 2008) and was reported from the Urals and in the mountains of southern Siberia and the Russian Far East. It has a large distribution area but its stations in Russia are at the northern limit of its distribution.

\section{Lobaria scrobiculata (Scop.) DC.}

Collecting site: 21,43 - on mossy stone.

Notes. Species new for Kodar Ridge. Previously reported from Delyun-Uransky Ridge by Bardunov et al. (2005).

\section{Lopadium pezizoideum (Ach.) Körb.}

Collecting Site: 18,39 - on mosses.

Notes. Species new for Kodar Ridge. Previously reported from Delyun-Uransky Ridge by Bardunov et al. (2005).

\section{Melanelia hepatizon (Ach.) A. Thell}

Collecting site: 4, 5, 6, 8, 10, 12, 17, 22, 27, 28, 29, $31,38,41,50,52,55,66-$ on stone and soil on stone.

Notes. Species new for Udokan Ridge. Previously reported from Delyun-Uransky (Bardunov et al. 2005) and Kodar Ridges (Makryi 2002; Bardunov et al. 2005).

\section{Melanelia stygia (L.) Essl.}

Collecting site: 1, 7, 15, 17, 22, 31, 41, 49, 50, 55 on stone and soil on stone.

Notes. Species new for Udokan Ridge. Previously reported from Delyun-Uransky (Bardunov et al. 2005) and Kodar Ridges (Makryi 2002; Bardunov et al. 2005).

!Melanelixia fuliginosa (Fr. ex Duby) O. Blanco et al.

Collecting SITE: $1,44,45$ - on stone.

!Melanelixia glabratula (Lamy) Sandler \& Arup COLLeCting Site: 44 - on bark of Betula sp. and Sorbus sibirica.

Melanohalea exasperatula (Nyl.) O. Blanco et al. Collecting Site: 19, 21, 43, 44, 45, 49, 66 - on bark of Sorbus sibirica, Pinus pumila and Betula sp., rotten wood, mossy stone, stone, plant debris.

Notes. Species new for Kodar Ridge. Previously reported from Delyun-Uransky by Bardunov et al. (2005) as Melanelia exasperatula (Nyl.) Essl.

!Micarea misella (Nyl.) Hedl.

COLleCting SITE: 46 - on rotten wood.

!Micarea nitschkeana (J. Lahm ex Rabenh.) Harm. COLLECTING SITE: 43 - on rotten wood.

\section{Micarea prasina Fr.}

COLLECTING SITE: 46 - on rotten wood.

Notes. Species new for Kodar Ridge. Previously reported from Yuzhno-Muyskiy and Kalar Ridges by Konoreva and Andreev (2013b).

!Miriquidica griseoatra (Flot.) Hertel \& Rambold COllecting SITE: 33 - on stone.

!Mycobilimbia pilularis (Körb.) Hafellner \& Türk COLLeCTING SITE: $43,44,47$ - on mosses, lignum, rotten branches and bark of Pinus pumila, and bark of Betula sp., 9 July 2013.

\section{Mycoblastus sanguinarius (L.) Norman}

COllecting site: 1, 21, 23, 31, 41, 49, 63 - on bark and branches of Pinus pumila, rotten wood, bark of Larix gmelinii.

Notes. Species new for Udokan Ridge. Previously reported from Delyun-Uransky (Bardunov et al. 2005) Kodar and Kalar Ridges (Konoreva \& Andreev 2013a, b). 
!Myriospora smaragdula (Wahlenb. ex Ach.)

Nägeli ex Uloth

Collecting site: 22 - on stone.

Nephroma bellum (Spreng.) Tuck.

Collecting site: 44 - on bark of Betula sp.

Notes. Species new for Kodar Ridge. Previously reported from Delyun-Uransky Ridge by Bardunov et al. (2005).

Nephroma isidiosum (Nyl.) Gyeln.

Collecting site: 43 - on mossy stone.

Notes. Species new for Kodar Ridge. Previously reported from Delyun-Uransky Ridge by Bardunov et al. (2005).

\section{!Ochrolechia androgyna (Hoffm.) Arnold}

Collecting site: 41 - on plant debris.

!Parmelina quercina (Willd.) Hale

Collecting site: 44 - on bark of Sorbus sibirica.

Peltigera collina (Ach.) Schrad.

Collecting Site: $13,21,44,45,72$ - on mossy stone, soil, mosses and bark of Populus sp.

Notes. Species new for Kodar Ridge. Previously reported from Delyun-Uransky and YuzhnoMuyskiy Ridges by Bardunov et al. (2005) and Konoreva and Andreev (2013b) respectively.

!Peltigera neopolydactyla (Gyeln.) Gyeln.

Collecting SITE: 43, 44 - on soil and mossy stone.

!Peltigera polidactylon (Neck.) Hoffm.

Collecting site: $21,44,61$ - on mosses and soil.

Peltigera praetextata (Flörke ex Sommerf.) Zopf Collecting site: $21,42,44,45,46,47$ - on mosses, soil, lignum and mossy stone.

Notes. Species new for Kodar Ridge. Previously reported from Delyun-Uransky Ridge by Bardunov et al. (2005).

\section{Peltigera venosa (L.) Hoffm.}

Collecting site: 44 - on mossy stone.

Notes. Species new for Kodar Ridge. Previ- ously reported from Delyun-Uransky Ridge by Bardunov et al. (2005).

Pertusaria dactylina (Ach.) Nyl.

Collecting site: 37,45 - on stone and mosses.

Notes. Species new for Kodar Ridge. Previously reported from Yuzhno-Muyskiy Ridge by Konoreva and Andreev (2013b).

\section{Pertusaria trochiscea Norman}

Collecting site: $42,44,45,46$ - on mosses, plant debris and mossy stone.

Notes. Species new for Kodar Ridge. Previously reported from Delyun-Uransky Ridge by Bardunov et al. (2005) as Pertusaria cf. trochiscea Norman.

!Phaeophyscia dissecta G. Urban., I. Urban. \& T. Otn.

Collecting site: 24,44 - on mossy stone.

Notes. This is a rare species in Russia. Previously reported from southern Siberia (Tuva, Buryatia, Trans-Baikal region) (Andreev et al. 2008; Urbanavichus 2010). It prefers coniferous and mixed coniferous-deciduous forests on warmed mountain slopes. It grows on moss-covered boulders and rocks.

!Phaeophyscia endococcinodes (Poelt) Essl.

Collecting site: 44,47 - on mossy stone.

Notes. This is a rare species in Russia. Previously reported from southern Siberia and the Russian Far East (Urbanavichus 2010). Reported from Asia, Africa, North and South America, Australia and New Zealand (Andreev et al. 2008). It grows on moss-covered rocks, rarely on mossy soil or old wood, in mountainous regions of southern latitudes.

\section{!Phaeophyscia hirtella Essl.}

Collecting site: 46,48 - on bark of Populus tremula L. and Salix sp.

Notes. This is a rare species in Russia. Previously reported from southern Siberia and the Russian Far East (Andreev et al. 2008; Urbanavichus 2010). Elsewhere, reported only from North 
America (Andreev et al. 2008; Hodkinson \& Case 2008). Usually it grows on trees with smooth bark in coniferous-deciduous, small-leaved and mixed forests.

\section{!Phaeophyscia orbicularis (Neck.) Moberg}

Collecting site: 44,72 - on mossy stone and bark of Populus sp.

!Phaeophyscia pusilloides (Zahlbr.) Essl.

Collecting SITE: 44 - on bark of Sorbus sibirica.

!Phaeorrhiza sareptana (Tomin) H. Mayrhofer \& Poelt

Collecting Site: 24 - on soil.

!Physcia tenella (Scop.) DC.

Collecting Site: 44 - on bark of Betula sp.

Physconia detersa (Nyl.) Poelt

Collecting Site: $43,44,45$ - on mossy stone.

!Physconia enteroxantha (Nyl.) Poelt

Collecting Site: 45 - on mosses.

!Pilophorus robustus Th. Fr.

Collecting Site: 2,41 - on stone near creek.

Pilophorus strumaticus Nyl. ex Cromb.

Collecting site: $1,13,15,41$ - on stone.

Notes. This is the second record of this species for Russia. Previously reported from the TransBaikal region on Kalar Ridge (Konoreva 2013). A rare species, it was reported from Sweden, Norway and the British Isles (Ahti \& Stenroos 2013; Smith et al. 2009). It is an arctic-alpine taxon, growing on siliceous rocks, usually in moist, shaded places.

!Placidium lachneum (Ach.) B. de Lesd.

COllecting Site: 24 - on stone.

!Placopsis lambii Hertel \& V. Wirth

COllecting SITE: $19,45-$ on stone.

!Protoblastenia calva (Dicks.) Zahlbr.

COLleCting SITE: 44 - on stone.
!Protoblastenia incrustans (DC.) J. Steiner

Collecting site: 46 - on stone.

Protoparmelia badia (Hoffm.) Hafellner

Collecting Site: 10, 11, 12, 17, 22, 29 - on stone.

Notes. Species new for Udokan Ridge. Previously reported from Delyun-Uransky and Kodar Ridges by Bardunov et al. (2005) and Makryi (2002).

Psora globifera (Ach.) A. Massal.

COllecting site: $24,44,45,54,69$ - on mosses and soil on stone.

Notes. Species new for Kodar and YuzhnoMuyskiy Ridges. Previously reported from Delyun-Uransky Ridge by Bardunov et al. (2005).

\section{!Psora vallesiaca (Schaer.) Timdal}

Collecting site: 45 - on soil on stone.

Notes. This is a rare species in Russia. Previously reported from the Arctic, eastern and southern Siberia (Urbanavichus 2010). Reported from Europe, North Africa, western Greenland and North America (Andreev et al. 2008; Kristinsson et al. 2010). It grows on calcareous soil.

Ramalina pollinaria (Westr.) Ach.

COLlecting SITE: 44,45 - on stone.

Notes. Species new for Kodar Ridge. Previously reported from Delyun-Uransky and YuzhnoMuyskiy Ridges by Bardunov et al. (2005) and Konoreva and Andreev (2013b) respectively.

Ramboldia elabens (Fr.) Kantvilas \& Elix Collecting Site: 20, 36, 43 - on lignum.

Notes. Species new for Kodar Ridge. Previously reported from Delyun-Uransky Ridge by Bardunov et al. (2005) as Pyrrhospora elabens (Fr.) Hafellner.

\section{!Rhizocarpon cinereonigrum Vain.}

Collecting site: $4,5,33$ - on stone.

Notes. This is a rare species in Russia. Previously reported from Novaya Zemlya (Kristinsson et al. 2010). A rare species, it was reported from Europe, North America and Greenland (Andreev et al. 2003). 
!Rhizocarpon copelandii (Körb.) Th. Fr.

COlleCting SITE: 47 - on stone.

Rhizocarpon eupetraeoides (Nyl.) Blomb. \& Forssell

Collecting Site: $1,4,10,12,17,22,27,31,38$ - on stone.

Notes. Species new for Udokan Ridge. Previously reported from Delyun-Uransky Ridge and Kodar Ridge by Bardunov et al. (2005).

!Rhizocarpon grande (Flörke) Arnold

COllecting SITE: 41,50 - on stone.

Rhizoplaca melanophthalma (DC.) Leuckert $\&$ Poelt

Collecting site: 13,26 - on stone.

Notes. Species new for Kodar Ridge. Previously reported from Delyun-Uransky Ridge by Bardunov et al. (2005).

Rhizoplaca subdiscrepans (Nyl.) R. Sant.

Collecting site: 6,24 - on stone.

Notes. Species new for Kodar and YuzhnoMuyskiy Ridges. Previously reported from Kalar Ridge by Konoreva and Andreev (2013b).

\section{!Rinodina conradii Körb.}

Collecting SITE: 44, 46, 47 - on bark of Sorbus sibirica and rotten wood.

!Rinodina mniaraea (Ach.) Körb.

Collecting site: 39 - on mosses.

!Rinodina pyrina (Ach.) Arnold

Collecting Site: 44 - on bark and rotten branches of Betula sp.

\section{Rinodina septentrionalis Malme}

Collecting site: $2,44,45,46,47,48,59,65$ - on lignum and on bark of Betula sp., Populus sp., P. tremula, Sorbus sibirica, Salix sp. and Pinus pumila.

Notes. Species new for Kodar Ridge. Previously reported from Delyun-Uransky Ridge by Bardunov et al. (2005).
!Rinodina turfacea var. cinereovirens (Vain.)

H. Mayrhofer

Collecting Site: 44 - on bark of Betula sp.

Rusavskia sorediata (Vain.) S. Y. Kondr. \& Kärnefelt

Collecting Site: $19,21,24,44,46$ - on stone.

Notes. Species new for Yuzhno-Muyskiy Ridge. Previously reported from Kodar Ridge by Makryi (2002) as Xanthoria sorediata (Vain.) Poelt.

Scoliciosporum chlorococcum (Graewe ex Stenh.) Vězda

COLleCting SITE: 44, 45, 46 - on bark of Sorbus sibirica and Betula sp., rotten wood.

Notes. Species new for Kodar Ridge. Previously reported from Delyun-Uransky Ridge by Bardunov et al. (2005).

Scoliciosporum umbrinum (Ach.) Arnold

COLLeCting Site: 1, 44, 46 - on bark of Sorbus sibirica and stone.

Notes. Species new for Kodar Ridge. Previously reported from Delyun-Uransky Ridge by Bardunov et al. (2005).

!Solorina bispora Nyl.

COllecting Site: 42 - on soil and mosses.

!Solorina saccata (L.) Ach.

Collecting SITE: 44 - on mossy stone and soil.

!Squamarina cartilaginea (With.) P. James

Collecting Site: 45 - on soil on stone.

Notes. This is a rare species in Russia. Previously reported from the Caucasus, Siberian Arctic and southern Siberia (Urbanavichus 2010). Reported from the British Isles, Europe, Macaronesia, North America, Asia and Africa (Smith et al. 2009). It grows on soil, mosses and hard calcareous rocks, preferring crevices of limestone cliffs and pavements, also on calcareous dunes and serpentine.

Stereocaulon subcoralloides (Nyl.) Nyl.

COLleCting SITE: 22 - on stone. 
Notes. Species new for Udokan Ridge. Previously reported from Kodar Ridge (Makryi 2002; Bardunov et al. 2005; Konoreva \& Andreev 2013a).

\section{!Stereocaulon wrightii Tuck.}

COllecting SITE: 23 - on stone.

Notes. This is a rare species in Russia. Previously reported from Chukotka (Kristinsson et al. 2010), Kamchatka, the Magadan region, Khabarovsk and Primorsky Krai (Dombrovskaya 1996). A rare species, it was reported from Japan (Honshu Island) (Dombrovskaya 1996) and North America (Alaska) (Weber \& Viereck 1967).

!Usnea hirta (L.) Weber ex F. H. Wigg.

Collecting site: 46 - on lignum.

Vulpicida pinastri (Scop.) J.-E. Mattsson

\& M. J. Lai

Collecting site: $13,21,24,31,36,43,44,45,46,49$, $50,56,58,59,61,65,68,71-$ on plant debris, lignum, bark of Betula ermanii, Salix sp., Larix gmelinii, Pinus pumila, Betula nana, Rhododendron aureum.

Notes. Species new for Yuzhno-Muyskiy Ridge. Previously reported from Kodar (Makryi 2002; Bardunov et al. 2005; Konoreva \& Andreev 2013a) and Delyun-Uransky Ridges (Bardunov et al. 2005).

\section{!Xanthomendoza ulophyllodes (Räsänen)}

Søchting et al.

Collecting Site: 45, 46 - on bark of Populus tremula.

Xanthoparmelia stenophylla (Ach.) Ahti

\& D. Hawksw.

Collecting site: $1,3,19,24,44,45$ - on stone and soil on stone.

Notes. Species new for Yuzhno-Muyskiy Ridge. Previously reported from Kodar (Makryi 2002; Bardunov et al. 2005) and Delyun-Uransky Ridges (Bardunov et al. 2005) as Xanthoparmelia somloënsis (Gyeln.) Hale.

\section{!Xylographa trunciseda (Th. Fr.) Minks ex Redinger}

COLleCting SITE: 46 - on rotten wood.
Notes. This is a rare species in Russia. Previously reported from the Southern Urals (Urbanavichene et al. 2013) and the Siberian Arctic (Urbanavichus 2010). Reported from Europe, Atlantic Maritime Canada, western North America and Asia (Smith et al. 2009; Spribille et al. 2014). It grows on slowly decaying wood in moist conditions.

Acknowledgements. We thank Dmitry Himelbrant (St. Petersburg, Russia) and Ivan Frolov (Yekaterinburg, Russia) for helping identify selected specimens, and the anonymous reviewers for helpful remarks and suggestions on the manuscript. The study was financially supported by the Russian Foundation for Basic Research (grants 14-04-01411, 14-04-10091 and 15-04-05971).

\section{REFERENCES}

AnONYmous 2008. Krasnaya kniga Rossiyskoy Federatsii (rasteniya i griby) - Red Data Book of the Russian Federation (Plants and fungi). KMK Scientific Press Ltd., Moscow (in Russian).

Ahti T. \& Stenroos S. 2013. Pilophorus. In: T. Ahti, S. StenRoos \& R. MOBerg (eds), Nordic Lichen Flora. 5: 87-89. Museum of Evolution, Uppsala University, Uppsala.

Andreev M. P., Bredkina L. I., Golubkova N. S., Dobrysh A. A., Kotlov Yu. V., MaKarova I. I., Urbanavichene I. N. \& Urbanavichus G. P. 2003. Handbook of the lichens of Russia 8. Bacidiaceae, Catillariaceae, Lecanoraceae, Megalariaceae, Mycobilimbiaceae, Rhizocarpaceae, Trapeliaceae. Nauka, St. Petersburg (in Russian).

Andreev M. P., Dobrysh A. A., Golubkova N. S., Himelbrant D. E., Kataeva O. A., Kotlov Yu. V., MaKarova I. I., Titov A. N., Tolpysheva T. YU., Urbanavichene I. N. \& Urbanavichus G. P. 2008. Handbook of the lichens of Russia 10. Agyriaceae, Anamylopsoraceae, Aphanopsidaceae, Arthrorhaphidaceae, Brigantiaeaceae, Chrysotrichaceae, Clavariaceae, Ectolechiaceae, Gomphillaceae, Gypsoplacaceae, Lecanoraceae, Lecideaceae, Mycoblastaceae, Phlyctidaceae, Physciaceae, Pilocarpaceae, Psoraceae, Ramalinaceae, Stereocaulaceae, Vezdaeaceae, Tricholomataceae. Nauka, St. Petersburg (in Russian).

Bardunov L. V., Chechetcina L. G., Makryi T. V., Malysheva L. I., Petrov A. N., Lishtva A. V., Lopatovskaya O. G. \& Maksimova E. N. 2005. The biota of the Vitimsky Reserve: Flora. Academic Publishing House "Geo", Novosibirsk (in Russian).

Brodo I. M., Sharnoff S. \& Sharnoff S. 2001. Lichens of North America. Yale University Press, New Haven \& London. 
Davydov E. A. 2014. The first checklist of lichens, lichenicolous, and allied fungi of Altaisky Krai (Siberia, Russia). Mycotaxon. 127. http://www.mycotaxon.com/resources/ checklists/davydov-v127-checklist.pdf.

DombrovskaYA A. V. 1996. Genus Stereocaulon in the USSR. Mir i Semya-95 Publ., St. Petersburg (in Russian).

Foucard T. 2001. Svenska skorplavar och svampar som växer på dem. Stenströms bokförlag/Interpublishing, Stockholm.

Gerasimova Yu. V., Konoreva L. A. \& Chesnokov S. V. 2014. The first record of Bacidia reagens (Ramalinaceae) for Eurasia. Novosti Sist. Nizsh. Rast. 48: 230-232.

Hodkinson B. P. \& Case M. A. 2008. A lichen survey of Williamsburg, Virginia. Banisteria 31: 24-30.

JøRGENSEN P. M. 1998. What shall we do with the blue-green counterparts? Lichenologist 30(4-5): 351-356.

Konoreva L. A. 2013. Pilophorus strumaticus (Cladoniaceae), a new lichen species for Russia. Novosti Sist. Nizsh. Rast. 47: 222-224 (in Russian with English summary).

Konoreva L. A. \& ANDreev M. P. 2013a. Lichens of mountain tundra of the Kodar Ridge. In: Modern botany in Russia. Proceedings of the XIII Congress of Russian Botanical Society and the conference "Scientific basis for the protection and management of vegetation cover of the Volga basin." V. 1: Embryology. Structural botany. Algologia. Mycology. Lichenology. Muscology. Paleobotany. Biosystematics. September 16-22, 2013, pp. 194-195. Tol'yatti, Cassandra (in Russian).

Konoreva L. A. \& Andreev M. P. 2013b. New lichen species in flora of eastern part of Stanovoye Upland (Transbaikalian Region, Russia). In: Biodiversity of the far north Ecosystems: Inventory, monitoring, protection: Reports of the II Russian Scientific Conference, 3-7 June, 2013, pp. 191-200. Institute of Biology, Komi Scientific Centre, Syktyvkar (in Russian with English summary).

Kristinsson H., Zhurbenko M. \& Hansen E. S. 2010. Panarctic checklist of lichens and lichenicolous fungi. CAFF Technical Report No. 20. CAFF International Secretariat, Akureyri, Iceland.

Kulakov V. S., Ryzhiy V. S. \& Snegur A. E. 2002. Geography of Kalarsky district. Poisk, Chita (in Russian).

Lishtva A. V., Himelbrant D. E. \& Stepanchikova I. S. 2013. Parmelia asiatica (Parmeliaceae): the first record for the lichen flora of Russia. Novosti Sist. Nizsh. Rast. 47: 225-229.

Lücking R., SÉrusiaux E. \& VĕZda A. 2005. Phylogeny and systematics of the lichen family Gomphillaceae (Ostropales) inferred from cladistic analysis of phenotype data. Lichenologist 37(2): 123-170.

Lücking R., Aptroot A., Umaña L., Chaves J. L., Sipman H. J. M. \& Nelsen M. P. 2006. A first assessment of the Ticolichen biodiversity inventory in Costa Rica: the genus
Gyalideopsis and its segregates (Ostropales: Gomphillaceae), with a world-wide key and name status checklist. Lichenologist 38(2): 131-160.

MAKRYI T. 1999. Lichens from Baikal region (Siberia) new for Russia. Cryptogamie, Mycol. 20(4): 329-334.

MAKrYI T. V. 2002. To the lichen flora of the Stanovoye Nagorye Uplands (Baikalian Siberia). I. The epilithic lichens of the Kodar Range. Turczaninowia 1(5): 47-67 (in Russian with English summary).

MAKRYI T. V. 2005. To the lichen flora of the Stanovoye Nagorye Uplands (Baikalian Siberia). II. The additions to the flora of epilithic lichens of the Kodar Range. Turczaninowia 8(3): 60-66 (in Russian with English summary).

MAKRYI T. V. 2012. Leptogium saxatile (Collemataceae), a new lichen species from Baikal Siberia, and two Leptogium species new for Mongolia. Novosti Sist. Nizsh. Rast. 46: 164-173 (in Russian with English summary).

Makryi T. V. 2013. Collema texanum (Collemataceae) - new lichen for Russia, and other saxicolous Collema species from Stanovoye Upland (Baikal Siberian Region). Novosti Sist. Nizsh. Rast. 47: 230-236 (in Russian with English summary).

Makryi T. V. 2014. A review of the sect. Mallotium of the genus Leptogium (Collemataceae) in Russia and L. pseudopapillosum and L. hirsutum new records for Russia. Novosti Sist. Nizsh. Rast. 48: 264-290 (in Russian with English summary).

Øvstedal D. O., Tønsberg T. \& ElvebakK A. 2009. The lichen flora of Svalbard. Sommerfeltia 33: 1-393.

Santesson R., Moberg R., Nordin A., Tønsberg T. \& VitiKAINEN O. 2004. Lichen-forming and lichenicolous fungi of Fennoscandia. Museum of Evolution, Uppsala University, Uppsala.

Sedel'nikova N. V. 2001. Lichens of West and East Sayans. Izdatel'stvo SO RAN, Novosibirsk (in Russian).

Smith C. W., Aptroot A., Coppins B. J., Fletcher A., GilBERT O. L., JAMES P. W. \& Wolseley P. A. (eds) 2009. The Lichen Flora of Great Britain and Ireland. The British Lichen Society, London.

Søchting U. \& Figueras G. 2007. Caloplaca lenae sp. nov., and other Caloplaca species with caloploicin and vicanicin. Lichenologist 39(1): 7-14.

Spribille T., Resl P., Ahti T., Pérez-Ortega S., Tønsberg T., Mayrhofer H. \& Lumbsch H. T. 2014. Molecular systematics of the wood-inhabiting, lichenforming genus $X y$ lographa (Baeomycetales, Ostropomycetidae) with eight new species. Symb. Bot. Upsal. 37(1): 1-87.

Stepanchikova I. S., Tagirdzhanova G. M. \& Himelbrant D. E. 2013. The lichens and allied fungi of the Smorodinka River Valley (Leningrad Region). Novosti Sist. Nizsh. Rast. 47: 262-278. 
TøNSBERG T. \& Goward T. 2001. Sticta oroborealis sp. nov., and other Pacific North American lichens forming dendriscocauloid cyanotypes. Bryologist 104(1): 12-23.

Urbanavichene I., Urbanavichus G., MeŽaka A. \& Palice Z. 2013. New records of lichens and lichenicolous fungi from the Southern Ural Mountains, Russia. II. Folia Cryptogamica Estonica 50: 73-80.
Urbanavichus G. 2010. A checklist of the lichen flora of Russia. Nauka, St. Petersburg (in Russian).

Weber W. A. \& Viereck L. A. 1967. Lichens of Mt. McKinley National Park, Alaska. Bryologist 70(2): 227-235.

Received 30 May 2015 